\title{
LncRNA SNHG15 regulates EGFR-TKI acquired resistance in lung adenocarcinoma through sponging miR-451 to upregulate MDR-1
}

\author{
Jiayuan Huang ${ }^{1}$, Banzhou Pan ${ }^{1}$, Guohao Xia', Jingni Zhu', Chenchen Li $^{1}$ and Jifeng Feng ${ }^{1}$
}

\begin{abstract}
Lung adenocarcinoma (LUAD) is the main component of non-small-cell lung cancer (NSCLC) and causes a great health concern globally. The top priority of LUAD treatment is to deal with gefitinib resistance. Long non-coding RNAs are certified to modify gefitinib resistance in the course of tumor aggravation. The study focuses on addressing the function of small nucleolar RNA host gene 15 (SNHG15) on modifying gefitinib resistance in LUAD. Previously, NOTCH pathway is implicated in LUAD chemo-resistance. SNHG15 level was boosted following the depletion of NOTCH-1 in A549/GR and H1975/GR cells. Functional studies indicated that SNHG15 and multidrug resistance protein 1 (MDR-1) were overexpressed and possess tumor-promoting functions in gefitinib-resistant LUAD cells while miR-451 was downregulated and possess tumor-suppressive behaviors in gefitinib-resistant LUAD cells. Mechanically, the SNHG15 was cytoplasmically distributed in GR LUAD cells. In addition, SNHG15 released MDR-1 from the suppression of miR451, leading to MDR-1 promotion. In addition, the elevation of SNHG15 could be attributed to ZEB1. Rescue assays highlighted that downstream molecules MDR-1 and miR-451 could reverse the effects of SNHG15 downregulation on gefitinib-resistant LUAD cells. SNHG15 could alter chemo-resistance of LUAD cells to Gefitinib via regulating miR-451/ MDR-1, which could be inspiring findings for the advancement of chemo-therapies for LUAD.
\end{abstract}

\section{Introduction}

Lung cancer is identified as one of the most malignant tumors, and causes countless deaths annually around the world ${ }^{1}$. Lung adenocarcinoma (LUAD) is the main class of non-small-cell lung cancer (NSCLC). In the last few decades, although depressing survival rate LUAD patients has been to some extent improved by drug therapies, such as the clinical applications of paclitaxel, docetaxel, gemcitabine, and vinorelbine, the poor prognosis remains a problem due to tumor metastasis or spread ${ }^{2}$. Therefore, it is a serious challenge for us to explore the mechanisms of drug resistance in LUAD, clarify the interactions among key regulatory targets, develop effective therapy methods,

Correspondence: Jifeng Feng (jifeng_feng@163.com)

'Department of Medical Oncology, Jiangsu Cancer Hospital \& Jiangsu institute of Cancer Research \& the Affiliated Cancer Hospital of Nanjing Medical

University, Nanjing, Jiangsu 210009, China

These authors contributed equally: Jiayuan Huang, Banzhou Pan

Edited by A. Stephanou and find important ways to improving and reversing drug resistance in the field of oncology research.

In previous studies, we have confirmed that $\mathrm{NOTCH}-1$, a major receptor in the $\mathrm{NOTCH}$ signaling pathway, affects the development of lung cancers ${ }^{3,4}$. Furthermore, the expression of $\mathrm{NOTCH}-1$ is closely related to histopathological typing, clinical stage, differentiation, metastasis, and recurrence in clinical specimens of $\mathrm{LUAD}^{5}$. In addition, the researching significance of $\mathrm{NOTCH}$ pathway has been demonstrated in other cancers, such as breast cancer $^{6}$, gastric cancer ${ }^{7}$, and esophageal carcinoma ${ }^{8}$.

Long non-coding RNAs (lncRNAs) are a cluster of noncoding RNAs (ncRNAs) with over 200 nucleotides and cannot code protein, which have drawn much attention in last 10 years ${ }^{9}$. Also, the lncRNAs with aberrant expression usually play a key role in regulating cancers, including LUAD. For illustration, lncRNA XIST facilitates metastasis and regulates EMT process in colorectal cancer ${ }^{10}$. LncRNA CRNDE/PRC2 gets involved in the radiotherapy

\section{(c) The Author(s) 2020}

(c) (i) Open Access This article is licensed under a Creative Commons Attribution 4.0 International License, which permits use, sharing, adaptation, distribution and reproduction c. in any medium or format, as long as you give appropriate credit to the original author(s) and the source, provide a link to the Creative Commons license, and indicate if changes were made. The images or other third party material in this article are included in the article's Creative Commons license, unless indicated otherwise in a credit line to the material. If material is not included in the article's Creative Commons license and your intended use is not permitted by statutory regulation or exceeds the permitted use, you will need to obtain permission directly from the copyright holder. To view a copy of this license, visit http://creativecommons.org/licenses/by/4.0/. 
resistance of LUAD via modulating the expression of p $21^{11}$. Further, the carcinogenesis of lncRNA SNHG15 has been verified in cancers, for example, lncRNA SNHG15 promotes colon cancer through stabilizing the expression of transcription factor (TF) Slug ${ }^{12}$. And SNHG15 depletion represses cell proliferation and EMT via regulating the NF- $\mathrm{KB}$ signaling pathway in renal cell carcinoma $^{13}$. Intriguingly, relation between SNHG15 and chemoresistance has been suggested. For instance, SNHG15 was contributing factor for temosolomide resistance and 5-FU resistance ${ }^{14,15}$. Nevertheless, the molecular regulation mechanism of SNHG15 in gefitinib resistance (GR) LUAD has not been reported yet.

MicroRNAs (miRNAs) also belong to ncRNAs, and miRNAs exert vital function in cancers by interacting with lncRNAs, such as IncRNA HULC contributing liver cancer development by sequestrating miR-186 ${ }^{16}$, and lncRNA DGCR5 promoting LUAD progression through repressing miR-22-3p ${ }^{17}$. The expression of miR-451 was significantly downregulated in NSCLC tissues, and it was closely associated with lymph node metastasis and survival prognosis in previous researches ${ }^{18}$. Moreover, overexpression of miR-451 could inhibit the sensitization of lung cancer cells to cisplatin by post-transcriptional targeting inhibition of RAB14, and reverse the drug-resistant phenotype $^{19}$. Besides, the effects of miR-451 on regulating docetaxel-resistant cells in LUAD have been illustrated $^{20,21}$. Nevertheless, miR-451 and GR has never been associated in LUAD before.

Also, the molecular mechanism about that the NOTCH signaling pathway participates in the regulation of tumor resistance via modulating the expression of downstream ncRNA is not defined yet. Therefore, in this study, we aimed to explore the role of SNHG15 and its downstream mechanism in regulating EGFR-TKI chemoresistance phenotype formation in LUAD, which may provide some novel thoughts for LUAD treatment.

\section{Materials and methods \\ Cell culture}

LUAD cell lines A549 and H1975 cells (both EGFR WT), acquired commercially from Chinese Academy of Sciences (Shanghai, China), were allowed to grow in Dulbecco's modified Eagle's medium (DMEM) with 5\% $\mathrm{CO}_{2}$ in air at $37^{\circ} \mathrm{C} .10 \%$ fetal bovine serum (FBS; HyClone, Logan, UT, USA) and double-antibiotics were utilized for cell culture. The Gefitinib-resistant A549 and H1975 cells (A549/GR and H1975/GR) were established in our clinical oncology laboratory center. Briefly, cells were subjected to continuous exposure to accumulating gefitinib (Sigma-Aldrich, St. Louis, USA) concentrations from 0.01 to $1 \mu \mathrm{M}$ for about 6 months, and then the established resistant cells underwent culture for 5 days in the gefitinib-free mediums prior to later experiments.

\section{RNA extraction and qRT-PCR}

Cellular total RNA was extracted from parent or gefitinib-resistant A549 and H1975 cells as per the guidelines of Trizol (Invitrogen, Carlsbad, CA, USA), and then was reverse-transcribed into cDNA as template for RNA detection. qRT-PCR was run with SYBR Premix EX Taq II (Takara Biotechnology, Tokyo, Japan) on Mx3000P qPCR system (Agilent Technologies, Santa Clara, CA, USA). The relative gene expression was calculated by $2^{-}$ $\Delta \Delta \mathrm{Ct}$ method, with GAPDH or U6 as reference gene.

\section{Western blot}

Cells were planted to 6-well plates prior to collection for western blotting. The cellular protein samples were obtained from RIPA buffer (Invitrogen), separated by $12 \%$ SDS-PAGE gels, and then transferred onto PVDF membranes. 5\% skimmed milk was applied to block membranes. Primary antibodies including anti-NOTCH-1 (ab52627), anti-E-cadherin (ab40772), anti-N-cadherin (ab98952), anti-Vimentin (ab92547), anti-ZEB1 (ab81972), anti-ZEB2 (ab138222), anti-EGFR (ab52894), anti-p-EGFR (ab40815), and anti-GAPDH (ab9484), as well as HRP-labeled IgG secondary antibodies, were purchased from Abcam (Cambridge, UK). Primary antibody anti-MDR-1 was obtained from Cell Signaling Technology (\#13978, Massachusetts, USA). After washing in phosphate-buffered saline (PBS), membranes were immersed in ECL Prime Western Blotting Detection reagent (GE Healthcare, Chicago, IL, USA) and observed in the dark.

\section{Cell transfection}

The specific shRNAs to NOTCH-1 (sh-NOTCH-1), SNHG15 (sh-SNHG15\#1/2), MDR-1 (sh-MDR-1\#1/2), ZEB1 (sh-ZEB1\#1/2), ZEB2 (sh-ZEB2\#1/2), and control shRNA (sh-NC), were all produced by RiboBio (Guangzhou, China). MiRNA mimics and NC mimics, miR-451 inhibitor and NC inhibitor, the pcDNA3.1/MDR-1, pcDNA3.1/ZEB1, pcDNA3.1/ZEB2, and control vectors, were all designed by GenePharma (Shanghai, China). Cells transfection with indicated plasmids was carried out using Lipofectamine 2000 (Invitrogen) for $48 \mathrm{~h}$.

\section{Colony formation}

Cultured cells were collected and plated to 6-well plates for 14 days of incubation in presence of gefitinib. After fixation in $96 \%$ ethanol, colonies were stained with $0.5 \%$ crystal violet for observation.

\section{EdU staining}

Transfected cells in 96-well plates were cultivated with $50 \mu \mathrm{M}$ of EdU solution (Ribobio) for $3 \mathrm{~h}$ and $4 \%$ paraformaldehyde for $15 \mathrm{~min}$. Following treatment with $1 \times$ Apollo ${ }^{\circledR} 488$ fluorescent staining, nuclei were stained 
with DAPI in the dark. Proliferative cells were identified under fluorescent microscope (Leica, Wetzlar, Germany).

\section{Flow cytometry analyses}

For cell cycle analysis, cells were seeded into 6-well plates, and then trypsinized and centrifuged. After washing in PBS, cells were treated with $70 \%$ of ice-cold ethanol for $2 \mathrm{~h}$ all night. The cell cycle detection kit (BD Bioscience, San Jose, CA, USA) was added for $30 \mathrm{~min}$. For cell apoptosis analysis, cells in cold PBS were incubated with $100 \mu \mathrm{L}$ of $1 \times$ binding buffer and $5 \mu \mathrm{L}$ of Annexin V$\mathrm{PE}$ and 7AAD (BD Biosciences) for $15 \mathrm{~min}$ in the dark. Both were analyzed by FACS cytometry (BD Biosciences).

\section{Cell migration assay}

Cells in PBS were re-suspended in serum-free culture medium and then added into the upper chamber $(8 \mu \mathrm{m}$ pore size; Millipore, Billerica, MA, USA). 20\% FBScomplete medium was added to the lower chamber. The migrated cells to the lower face of the filters were subjected to methanol for fixation, crystal violet for staining, and microscope for imaging.

\section{Immunofluorescence (IF)}

Cells were pre-plated on the cultured slides for $24 \mathrm{~h}$, rinsed in PBS and then fixed with ice-cold methanolacetone for $10 \mathrm{~min}$. Following blocking with 5\% skimmed milk and detection with primary antibodies against $\mathrm{E}$ cadherin and $\mathrm{N}$-cadherin in PBS for $2 \mathrm{~h}$ at room temperature, secondary antibodies were added for $1 \mathrm{~h}$. The slides were dyed with DAPI for $10 \mathrm{~min}$ and tested by confocal imaging system (Olympus, Tokyo, Japan).

\section{Subcellular fractionation}

Nuclear and cytoplasmic RNAs were extracted in line with the invitrogen nuclear extraction protocol. Cell lysates in cell fractionation buffer were centrifuged to acquire cell cytoplasm. Then, cell disruption buffer was added for lying cell nuclei. The SNHG15 level was examined by qRT-PCR, with GAPDH and U6 as cytoplasmic and nuclear normalizations.

\section{Dual-luciferase reporter gene assay}

The sequence fragment of SNHG15 containing the putative target sites for miR-873-5p, miR-24-3p, miR-1533p, miR-451, miR-200a-3p or miR-141-3p were amplified and inserted into the pmirGLO Luciferase Vector, then cotransfected with miRNA mimics and NC mimics into cells. The pmirGLO-SNHG15-WT/MUT and pmirGLO-MDR1-WT/MUT reporter vectors were formed with the wildtype or mutant miR-451 binding sites. For promoter assay, cells were co-transfected with pGL3-reporter-vector containing SNHG15 promoter and ZEB1 overexpression or silencing vectors. All of the luciferase gene activities were assayed by dual-Luciferase reporter assay system (Promega, Madison, WI, USA).

\section{RNA immunoprecipitation (RIP)}

In light of the protocol of Magna RIP Kit (Millipore), RIP assay was conducted with human anti-Ago2 antibody or negative control normal mouse IgG antibody (Millipore). Cell lysates were incubated with magnetic beads conjugated with antibodies in RIP buffer. The finally precipitated RNAs were isolated for qRT-PCR.

\section{RNA pull-down}

The sequences of miR-451 containing possible SNHG15 binding sites or mutant sites were biotinylated into BiomiR-451-WT and Bio-miR-451-MUT. Lysates were mixed with biotin-labeled RNAs for $1 \mathrm{~h}$, with Bio-NC as control. After adding magnetic beads for $30 \mathrm{~min}$, qRTPCR was performed to measure RNA levels.

\section{Chromatin immunoprecipitation (ChIP)}

Cells in formaldehyde were sonicated for obtaining chromatin fragments of 200-500 bp. Immunoprecipitation was conducted with anti-ZEB1 and anti-IgG antibodies (Millipore). The finally retrieved chromatin DNA was subjected to qRT-PCR analysis.

\section{Xenograft models}

The BALB/c nude mice (male, 4-6 weeks) weighing 18-20 g were bought from Shanghai SLAC Laboratory Animal Co. Ltd. (Shanghai, China). Animal experiments obeyed guidelines of the Institutional Animal Care and Use Committee (IACUC). A549/GR cells $\left(5 \times 10^{6}\right)$ suspended in PBS $(50 \mu \mathrm{L})$ with transfection of sh-NC or shSNHG15\#1 were subcutaneously inoculated into right flanks of each mouse. Afterwards, mice randomly group in 4 , and 2 groups were treated with gefitinib $(50 \mathrm{mg} / \mathrm{kg})$ or DMSO control 3 days, one time for 4 weeks. Tumor generation was monitored with the vernier caliper once a week. To calculate tumor volume, following formula was applied: Volume $\left(\mathrm{mm}^{3}\right)=\left(\right.$ Length $\left.\times \mathrm{Width}^{2}\right) / 2$. Four weeks later, the mice were sacrificed and subsequent assays gained permission of the Animal Experimental Ethics Committee of Jiangsu Cancer Hospital.

\section{Statistical analysis}

Quantitative data were exhibited as mean \pm S.D. from three separated replications and analyzed by Prism 6.0 for Windows (GraphPad, San Diego, CA, USA). Significant differences between groups were compared with Student's $t$ test (two groups) or one-way ANOVA (multiple groups), with the threshold of $P<0.05$. 


\section{Results}

NOTCH signaling-related SNHG15 accelerates gefitinibresistant LUAD cell malignant behaviors

As annotated previously, $\mathrm{NOTCH}$ signaling pathway is related to EGFR-TKI resistance and the clinical significance of NOTCH1 has also been highlighted previously in $\mathrm{LUAD}^{3,4}$. We also interrogated the relevance of NOTCH1 signaling with GR in LUAD. IC $_{50}$ of A549/ GR cells and H1975/GR cells increased versus the parental A549 and H1975 cells, confirming the acquirement of GR in both cell lines (Fig. 1a). qRT-PCR assay detected NOTCH pathway-related gene expressions. Results indicated that in comparison to parental LUAD cells, GR LUAD cells (A549/GR and H1975/GR) presented an elevated mRNA level of NOTCH pathwayrelated genes including $\mathrm{NOTCH}-1$, NOTCH-2, NOTCH-3, NOTCH-4, Jagged-1, Jagged-2, and Delta-1, among which NOTCH1 expression was the most upregulated (Fig. 1b). Furthermore, western blot assay also confirmed that NOTCH1 was highly expressed in A549/GR and H1975/GR cells (Fig. 1c). In addition, previous studies have shown that $\mathrm{NOTCH}-1$ can regulate EGFR expression in lung cancer cells ${ }^{4,22}$. Given that geftinib is an EGFR-TKI, we detected the influence of NOTCH-1 on EGFR expression. Consistently, we confirmed that NOTCH-1 indeed decreased EGFR level in A549/GR and H1975/GR cells (Fig. S1a). Then, we tried to detect whether SNHG15 can be affected by NOTCH-1. We observed that SNHG15 level decreased upon NOTCH-1 silence in A549/GR and H1975/GR cells (Fig. 1d), indicating that SNHG15 might participate in $\mathrm{NOTCH}-1$ effect on GR in LUAD cells. Interestingly, we observed that SNHG15 knockdown failed to affect both total and phosphorylated EGFR levels in A549/GR and H1975/GR cells (Fig. S1b). Hence, we were interested whether SNHG15 could be a way for NOTCH-1 to regulate GR independent from EGFR signaling in GR LUAD cells. Hence, SNHG15 loss-of-function assay was conducted. Small hairpin RNAs against SNHG15 were constructed and transfected into A549/GR and H1975/ GR cells. SNHG15 level was reduced in A549/GR and H1975/GR cells following SNHG15 knockdown (Fig. 1e). As demonstrated, we discovered that colony formation efficiency of A549/GR and H1975/GR cells was impaired following SNHG15 depletion (Fig. 1f). Similarly, EdU-positive cells were also reduced in the absence of SNHG15 (Fig. 1g). In cell cycle detection, it showed that SNHG15 depletion arrested A549/GR and H1975/GR at G0/G1 stage, while cell ratio at S-phase declined upon SNHG15 silencing (Fig. 1h). Knockdown of SNHG15 increased the proportion of apoptotic A549/ GR and H1975/GR cells (Fig. 1i). Collectively, observations above highlighted that SNHG15 is crucial for GR in LUAD cells.

\section{Knockdown of SNHG15 retards the aggressiveness of gefitinib-resistant LUAD cells}

Next, we also probed the function of SNHG15 on gefitinib-resistant LUAD cell migration and EMT program. As indicated in Transwell migration assay, SNHG15 silencing prohibited migration of A549/GR and H1975/GR cells (Fig. 2a). In western blot analysis, Ecadherin was increased while Vimentin, N-cadherin, ZEB1, and ZEB2 declined after SNHG15 blockade, hinting that EMT was also hindered by SNHG15 silencing (Fig. 2b). Consistent with the data in western blot, IF also exhibited the increase of E-cadherin and decline of $\mathrm{N}$ cadherin following SNHG15 downregulation (Fig. 2c). Taken together, gefitinib-resistant LUAD cell migration and EMT were limited by the downregulation of SNHG15.

\section{SNHG15 sponges miR-451 in gefitinib-resistant LUAD cells}

Afterwards, SNHG15-mediated mechanism was detected. First, the subcellular presence of SNHG15 in A549/ GR and H1975/GR cells was explored. Data supported the distribution of SNHG15 majorly in A549/GR and H1975/ GR cell cytoplasm, indicating the possibility that SNHG15 possessed post-transcriptional regulatory function (Fig. 3a). Starbase (http://starbase.sysu.edu.cn/starbase2/ rbpMrna.php) predicted 13 miRNAs which could bind to SNHG15. Subsequently, to screen out the specific miRNA, the expressions of 13 miRNAs were compared in A549/GR and its parental cell by qRT-PCR. It turned out that 6 miRNAs (miR-873-5p, miR-24-3p, miR-153-3p, miR-451, miR-141-3p, and miR-200a-3p) were obviously downregulated (Fig. 3b). After that, these 6 miRNAs were upregulated via the transfection of miRNA mimics (Fig. 3c). Luciferase reporter assay suggested that only miR-451 mimics greatly suppressed SNHG15 luciferase activity in A549/GR and H1975/GR cells (Fig. 3d). Therefore, miR-451 was chosen. Binding sites between SNHG15 and miR-451 were acquired from online starbase (Fig. 3e). Based on this, luciferase reporter assay was performed to confirm the interaction between SNHG15 and miR-451. Results indicated that the activity of wildtype SNHG15 reporter was reduced by miR-451 mimics, while the activity of mutant SNHG15 reporter with mutations at the predicted miR-451 binding sites did not change in the presence of miR-451 mimics (Fig. 3f). RIP assay evidenced that both SNHG15 and miR-451 were enriched in the binding complex of the core protein of RNA-induced mediating complex, Ago2 (Fig. 3g). It was evident in RNA pull-down assay that Bio-miR-451-WT, but not Bio-NC or Bio-miR-451-MUT, precipitated SNHG15 (Fig. 3h). Afterwards, we monitored the function of miR-451 in A549/GR and H1975/GR cell growth, migration and EMT process. As presented in Fig. 3i, j and $\mathrm{S} 2 \mathrm{a}, \mathrm{b}$, colony formation and proliferation capacities of 


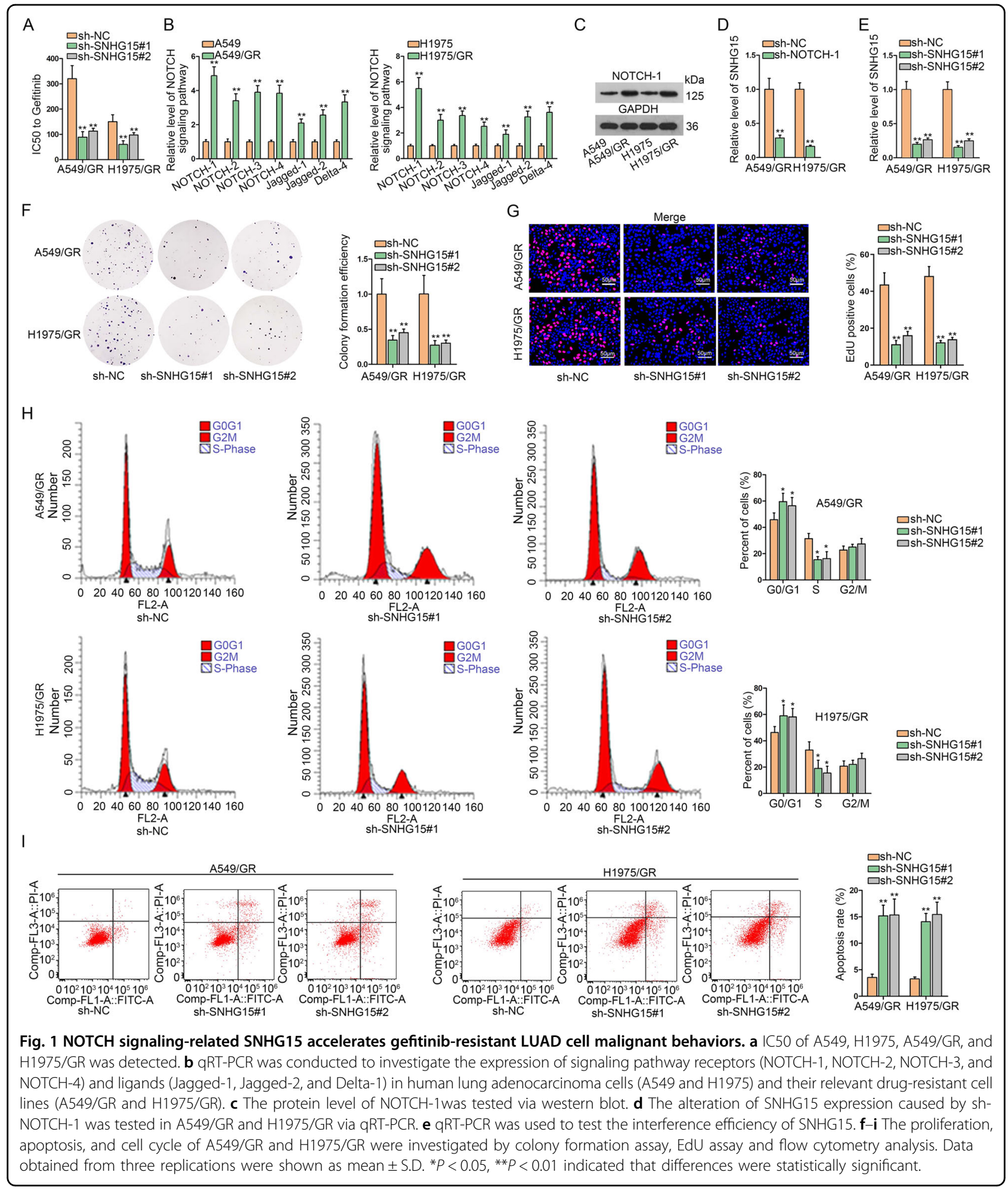

A549/GR and H1975/GR were restrained upon miR-451 overexpression. In contrast, A549/GR and H1975/GR cell apoptosis was accelerated compared with cells in NC group (Figs. 3k and S2c). The migration of cells was weakened by miR-451 mimic versus $\mathrm{NC}$ mimic (Figs. 31 and S2d). As for EMT program, we unveiled via western blot that augmentation of miR-451 hampered the process of EMT (Fig. 3m). In addition, a previous study argued that miR-451 overexpression resulted in repressed EGFR signaling in breast cancer ${ }^{23}$, so we detected whether miR- 


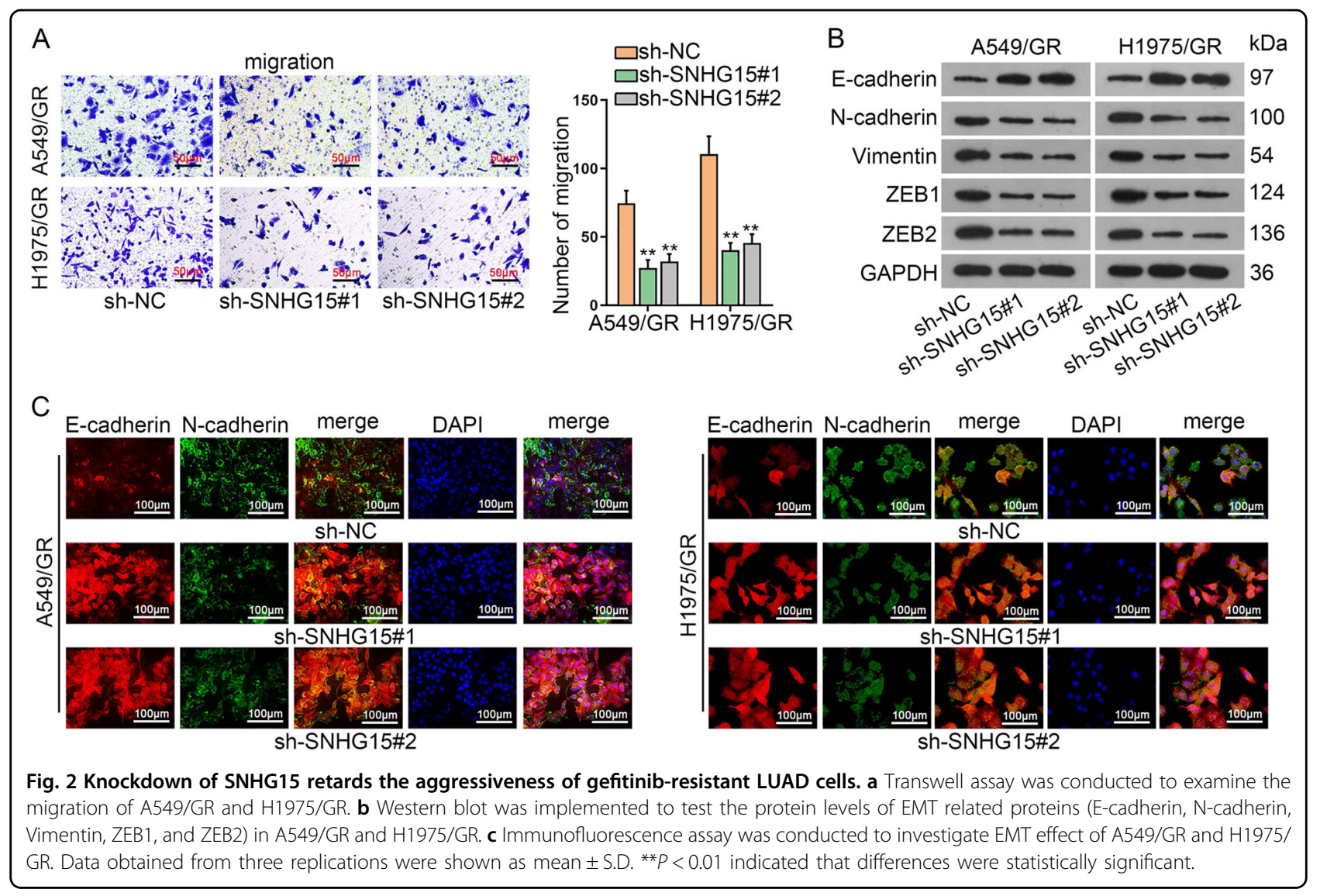

451 affected EGFR in LUAD cells as well. Unexpectedly, miR-451 mimic failed to alter p-EGFR and EGFR levels in A549/GR and H1975/GR cells (Fig. S2e). Altogether, SNHG15 acted as a sponge of miR-451, a tumor suppressor in gefitinib-resistant LUAD cells.

\section{SNHG15 facilitates MDR-1 expression through serving as a ceRNA for miR-451}

Bioinformatics analysis uncovered putative mRNAs for miR-451 and we profiled the expression of predicated mRNAs in H1975/GR and H1975 cells. Among top five overexpressed genes, MDR-1 (multidrug resistance protein 1) was the most overtly upregulated one in A549/GR versus A549 cells (Fig. 4a). Binding sites between MDR-1 and miR-451 were exhibited in Fig. 4 b. Luciferase reporter assay described that miR-451 mimics reduced the luciferase activity of MDR-1-WT, but miR-451 mimics did not change MDR-1-MUT (mutations at the miR-451 binding sites) activity (Fig. 4c). The modulation of SNHG15 and miR-451 on MDR-1 expression was discussed and miR-451 was downregulated by transfected miR-451 inhibitor into A549/GR cells (Fig. S2f). Subsequent qRT-PCR assay pointed out that sh-SNHG15\#1 suppressed MDR-1
mRNA but the introduction of miR-451 inhibitor reversed the impact. Similar trends could also be observed in western blot assay, which suggested that SNHG15-induced decline of MDR-1 protein was recovered by miR-451 inhibitor (Fig. 4d). In addition, whether MDR-1 functioned in gefitinib-resistant LUAD cells was corroborated. Firstly, MDR-1 was downregulated by shRNAs targeting MDR-1 (sh-MDR-1\#1 and sh-MDR-1\#2) (Fig. 4e). Colony formation ability of A549/GR and H1975/GR was attenuated by the loss of MDR-1 (Fig. 4f and S3a). Besides, the growth of A549/ GR and H1975/GR was also retarded via MDR-1 deficiency (Figs. $4 \mathrm{~g}$ and S3b). Conversely, the facilitating effect of MDR-1 downregulation on A549/GR and H1975/GR apoptosis was validated (Fig. 4h and S3c). Moreover, influenced by MDR-1 depletion, A549/GR and H1975/GR migration was suppressed (Figs. 4i and S3d). In western blot assay, we found that MDR-1 downregulation was able to attenuate EMT process in A549/GR and H1975/GR cells (Fig. 4j). However, the MDR-1 knockdown triggered no significant alteration in EGFR and p-EGFR levels in A549/GR and H1975/GR cells (Fig. S3e). These findings demonstrated that MDR- 


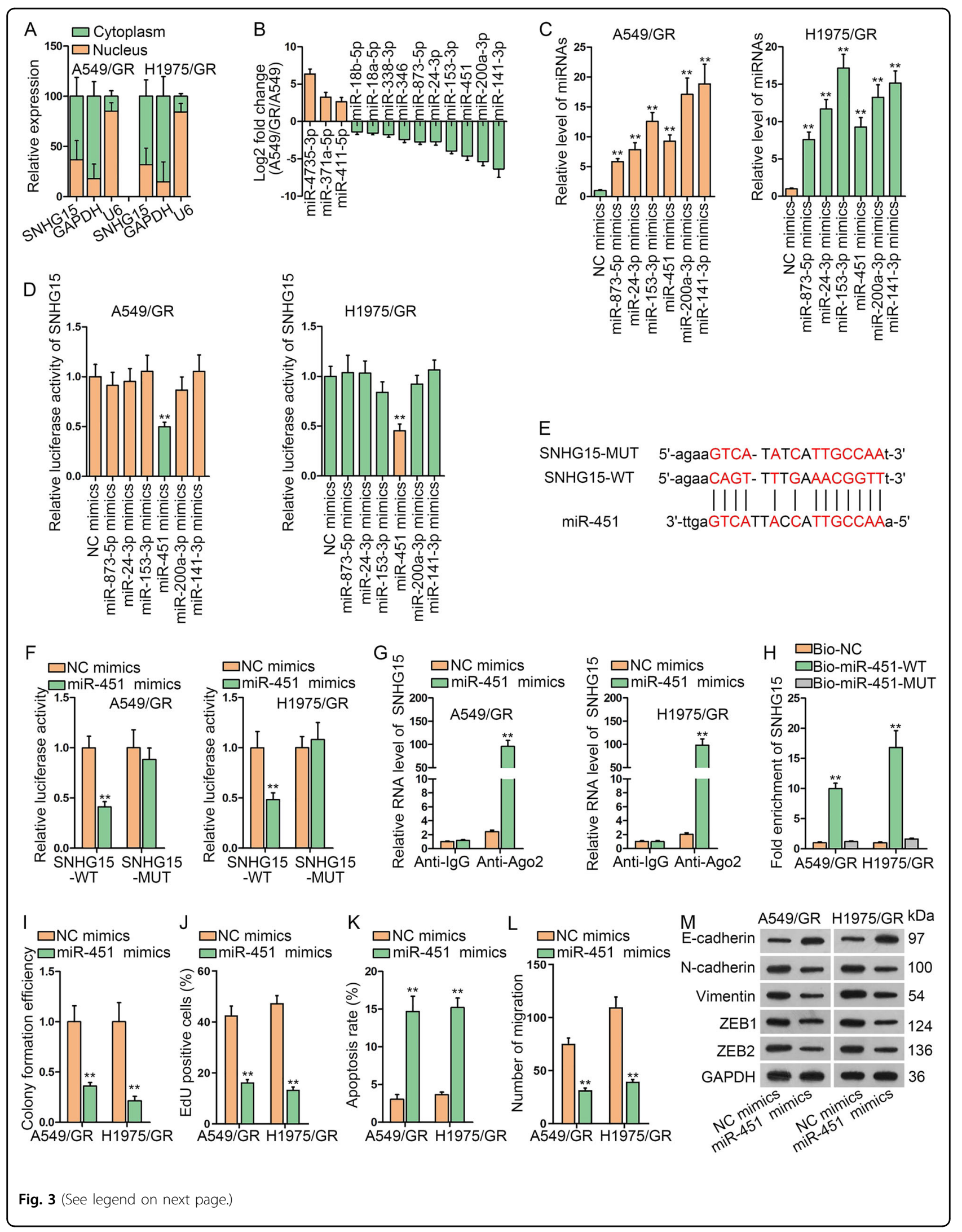


(see figure on previous page)

Fig. 3 SNHG15 sponges miR-451 in gefitinib-resistant LUAD cells. a Subcellular fraction assay was used to locate the position of SNHG15. $\mathbf{b}$ The fold change of 13 miRNAs in A549/GR versus A549 was detected to find potential miRNA. c The overexpression efficiency of 6 miRNAS (miR-873-5p mimics, miR-24-3p mimics, miR-153-3p mimics, miR-451 mimics, miR-200a-3p mimics, and miR-141-3p mimics) was tested by qRT-PCR in A549/GR and H1975/GR. d Luciferase reporter assay was conducted to find the suitable miRNA. e Bioinformatics tool was used for drawing the binding site between SNHG15 and miR-451, and SNHG15-MUT sequence logo was presented. $\mathbf{f}-\mathbf{h}$ Luciferase reporter assay, RIP assay and RNA pull-down assay were conducted to confirm the binding of SNHG15 with miR-451. i-k Colony formation assay, EdU assay and flow cytometry analysis were implemented to test the proliferation and apoptosis of A549/GR and H1975/GR when miR-451 was overexpressed. I Quantification of migrated cells in the transwell system under miR-451 overexpression. $\mathbf{m}$ The protein levels of EMT related proteins (E-cadherin, N-cadherin, Vimentin, ZEB1, and ZEB2) in A549/GR and H1975/GR were detected by western blot when miR-451 was overexpressed. Data obtained from three replications were shown as mean \pm S.D. ${ }^{* *} P<0.01$ indicated that differences were statistically significant.

1 was protected by SNHG15 against the suppression of $\operatorname{miR}-451$.

\section{MiR-451 or MDR-1 countervails the impacts of SNHG15 in gefitinib-resistant LUAD cells}

The ceRNA pathway of SNHG15/miR-451/MDR-1 was then functionally confirmed in cellular experiments. MDR-1 was ectopically expressed by transfecting pcDNA3.1/MDR-1 according to qRT-PCR (Fig. S3f). Consequently, the anti-proliferation property of shSNHG15\#1 could be counterbalanced by miR-451 inhibitor or pcDNA3.1/MDR-1 (Fig. 5a, b). Flow cytometry experiments manifested that sh-SNHG15\#1-caused apoptosis promotion could be alleviated by miR-451 inhibitor or pcDNA3.1/MDR-1 (Fig. 5c). A549/GR cell migration was retarded by sh-SNHG15\#1, while was later facilitated via the transfection of miR-451 inhibitor or pcDNA3.1/MDR-1 (Fig. 5d). As manifested in western blot assay, the suppression on EMT caused by SNHG15 deficiency was mitigated by miR-451 downregulation or MDR-1 overexpression (Fig. 5e). In conclusion, we further confirmed that SNHG15/miR-451/MDR-1 pathway conferred gefitinib-resistant LUAD cell growth, migration, and EMT.

\section{ZEB1 trans-activates SNHG15 expression in gefitinib- resistant LUAD cells}

We have validated that ZEB1 and ZEB2 can be positively regulated by SNHG15. Researches have supported that ZEB1 and ZEB2, recognized as TFs, can regulate transcription of target genes through binding to the promoter $^{24}$, and several studies unveiled that ZEB1 can upregulate lncRNAs through transactivation ${ }^{25,26}$. Therefore, we wondered whether ZEB1 and ZEB2 could regulate SNHG15 in LUAD. To begin with, we ectopically enforced or suppressed ZEB1 and ZEB2 expressions and verified the results by qRT-PCR (Fig. 6a, b). Subsequently, the influence of ZEB1 and ZEB2 on SNHG15 expression was investigated. Overexpression of ZEB1 led to an increased level of SNHG15 and ZEB1 knockdown had opposite impacts (Fig. 6c). Apparently, either overexpression or repression of ZEB2 was unable to alter
SNHG15 expression (Fig. 6d). JASPAR (http://jaspar. genereg.net/) provided the ZEB1 motif and potential sites (site 1, site 2, and site 3) in SNHG15 promoter for this interaction (Fig. 6e). Referring to ChIP experiments, in SNHG15 promoter was abundant in ZEB1 precipitates (Fig. 6f). In addition, we uncovered that luciferase activity of SNHG15 promoter was promoted in response to ZEB1overexpressing plasmids when site 1 or site 2 in SNHG15 promoter was mutated, while the activity of SNHG15 promoter with mutations at site 3 or the mutation at all three sites did not react to ZEB1 overexpression (Fig. 6g). To substantiate that site 3 in SNHG15 promoter was responsible for the interaction with ZEB1, we carried out another luciferase reporter assay. The findings supported that sh-ZEB1\#1 and sh-ZEB1\#2 reduced the activity of wild-type SNHG15 promoter, while the luciferase activity could not be altered by sh-ZEB1\#1 and sh-ZEB1\#2 when site 3 was mutated (Fig. 6h). In summary, ZEB1 transcriptionally accelerated SNHG15 expression in gefitinibresistant LUAD cells.

\section{SNHG15 contributed to GR in vivo}

Later, we unfolded animal experiments by injecting A549/GR cells into mice to generate in vivo xenografts. By monitoring tumor growth, we discovered that knockdown of SNHG15 slowed down the tumor growth of A549/GR cells in vivo (Fig. 7a). Also, gefitinib treatment repressed tumor growth, and knockdown of SNHG15 facilitated the effect of gefitinib (Fig. 7a). The tumor weight declined under SNHG15 deficiency or gefitinib treatment, and SNHG15 silence also further reduced the tumor weight that was decreased by gefitinib (Fig. 7b). Moreover, SNHG15 level and MDR-1 mRNA and protein levels declined in xenografts with SNHG15 knockdown in mice without or with gefitinib treatment (Fig. 7c, d). SNHG15 silence or gefitinib treatment caused the increase of E-cadherin and decrease of $\mathrm{N}$-cadherin, Vimentin, ZEB1, and ZEB2 levels, and SNHG15 silence also further strengthened the effect of gefitinib on the levels of these proteins in xenografts (Fig. 7d). Together, SNHG15 knockdown reversed $G R$ in vivo. 
A
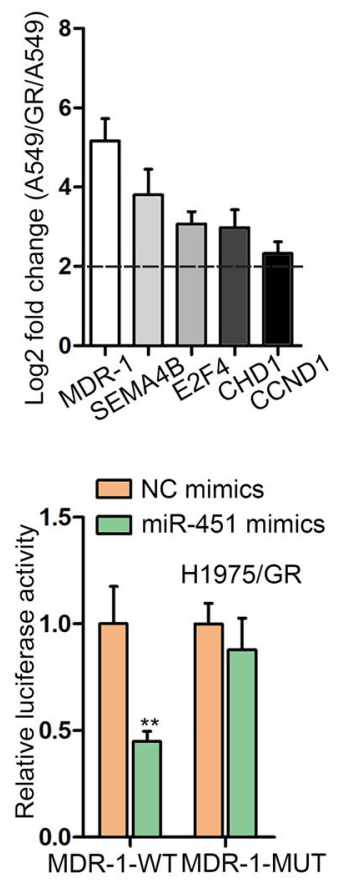

E $\square$ sh-NC

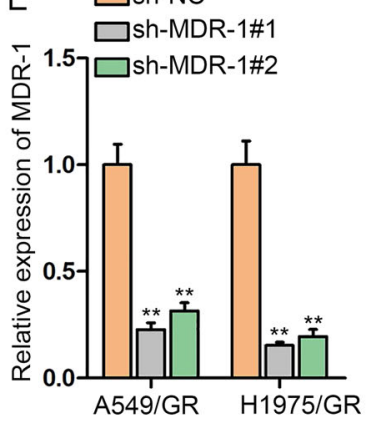

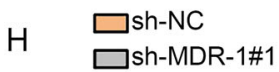

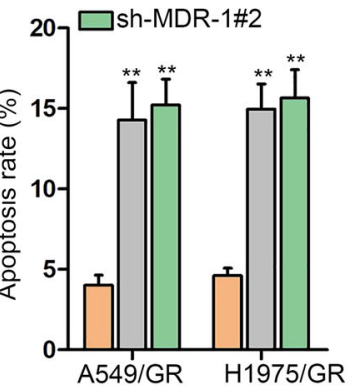

B

$\begin{array}{ll}\text { MODR1-WT } & \text { 5'TTCTCTCCCTTGGCATCCGGTTA 3' } \\ \text { MiR-451 } & \text { 3' UUGAGUCAUUACCAUUGCCAAA 5' } \\ \text { MODR1-WT } & \text { 5'TTCTCTCCCTTGGCATCGCCAAA 3' }\end{array}$

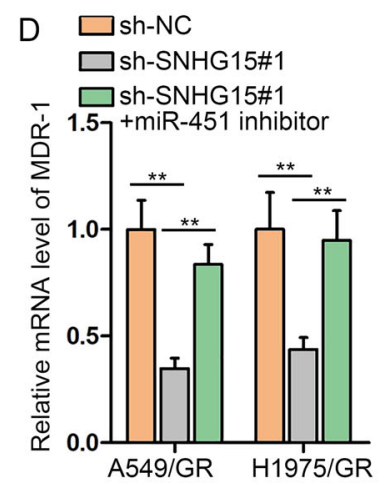

F $\square$ sh-NC
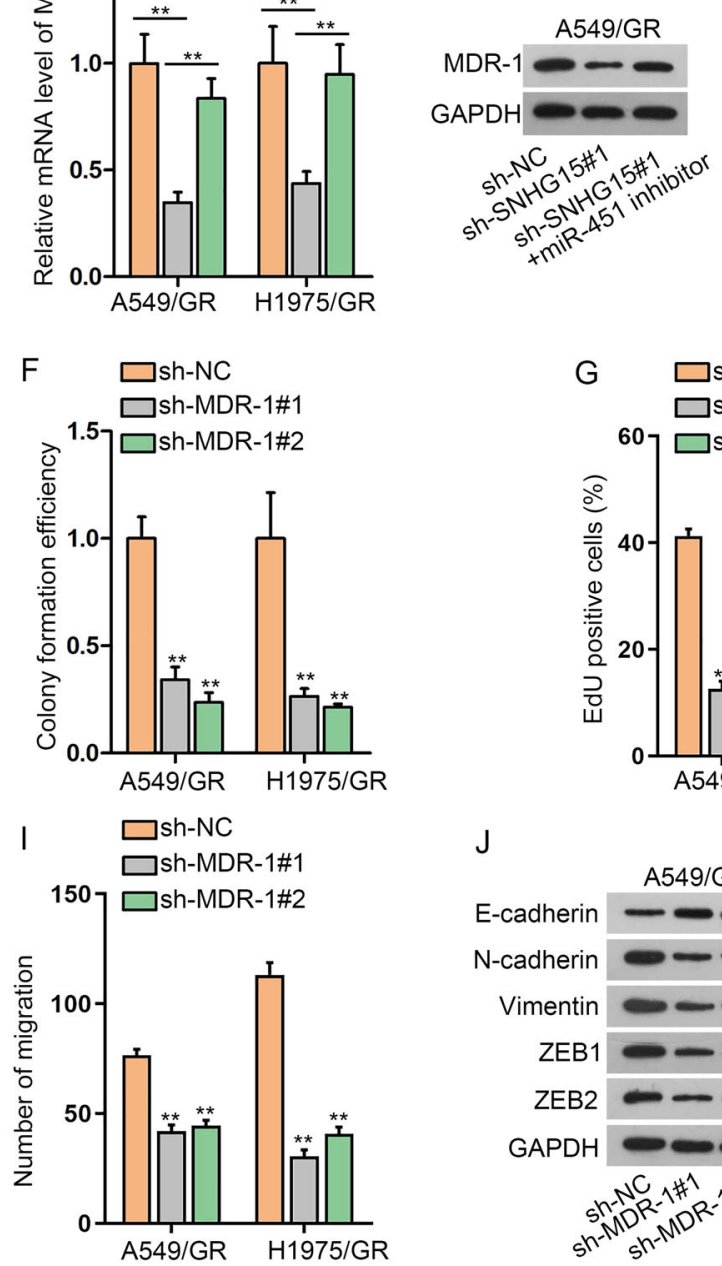

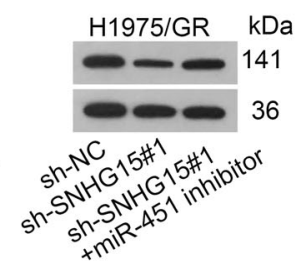

G $\square$ sh-NC
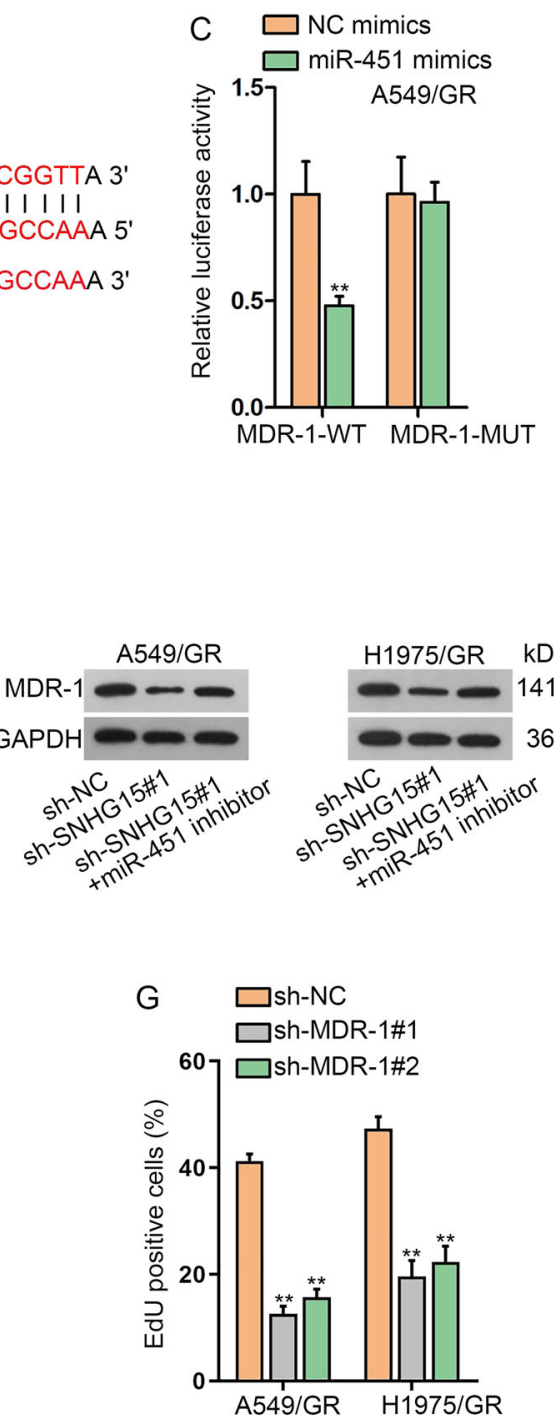

$\mathrm{J}$

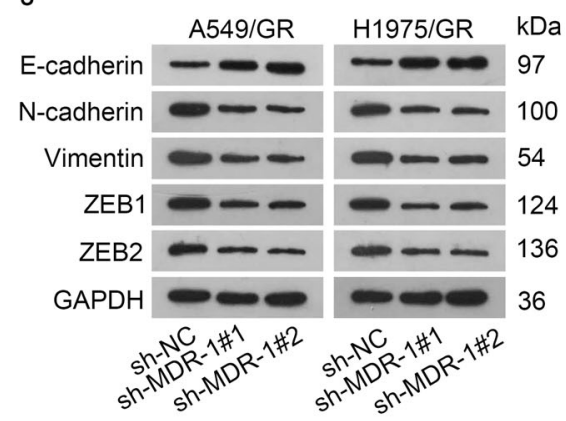

Fig. 4 SNHG15 facilitates MDR-1 expression through serving as a ceRNA for miR-451. a The fold change of 5 mRNAs whose expressions were most significantly upregulated in A549/GR versus A549 was detected for searching potential mRNA. $\mathbf{b}$ The binding site between miR-451 and MDR-1 was presented according to bioinformatics tool. c The binding relationships of miR-451/MDR-1 and miR-451/SNHG15 were confirmed by luciferase reporter assay. d Western blots of MDR-1 in A549/GR and H1975/GR with suggested transfections. e Inhibition efficiency of MDR-1 was tested by qRTPCR in A549/GR and H1975/GR. f-i Colony formation assay, EdU assay, flow cytometry analysis, and Transwell assay were conducted to test the proliferation, apoptosis and migration of A549/GR and H1975/GR under the influence of MDR-1 knockdown. $\mathbf{j}$ Western blot was used to test the protein levels of EMT related proteins (E-cadherin, N-cadherin, Vimentin, ZEB1, and ZEB2) in A549/GR and H1975/GR under the influence of inhibited MDR-1. Data obtained from three replications were shown as mean \pm S.D. ${ }^{* *} P<0.01$ indicated that differences were statistically significant. 


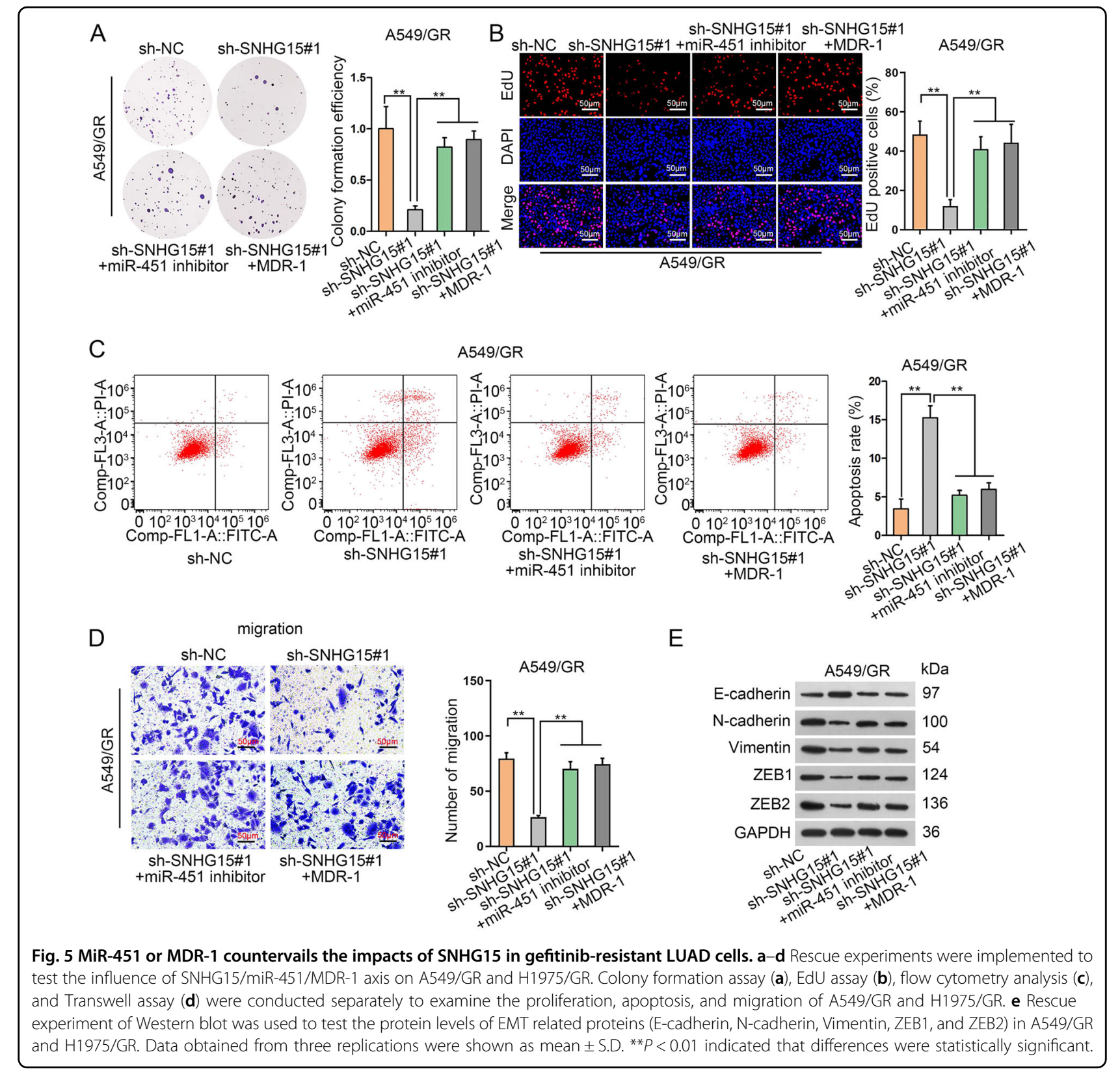

\section{Discussion}

Previous studies established that the employment of EGFR tyrosine-kinase inhibitors (EGFR-TKIs) (such as gefitinib, erlotinib and ectinib) have achieved encouraging progress in treating NSCLC harboring EGFR mutations, unfortunately, NSCLC patients would inevitably become drug resistant ${ }^{27-31}$. NOTCH-1 has been reported to promote GR of NSCLC cells and epithelial-mesenchymal transition (EMT) process in gefitinib-resistant NSCLC cells $^{3,4}$. NOTCH-1 was discovered to be upregulated in Hodgkin's lymphoma, and its mutations were spot to serve as secondary events in some T-cell acute lymphoblastic leukemia sufferers ${ }^{32,33}$. Besides, NOTCH-1 has also been elucidated as critical players in several cancers, such as glioma and prostate cancer ${ }^{34,35}$. In lung cancer, co-expression of NOTCH-1 and vascular endothelial growth factor-A predicts poor survival ${ }^{36}$. In NSCLC cells, rhamnetin and cirsiliol could suppress EMT process via miR-34a-mediated inhibitive effects on NOTCH-1 level ${ }^{37}$. In LUAD, NOTCH-1 could activate the IGF-1R pathway to promote survival of LUAD cells under hypoxic treatment $^{38}$. Although previous study illustrated that $\mathrm{NOTCH}-1$ was conspicuously downregulated in LUAD cells and tumor tissues compared with normal cells and tissues $^{5}$, former finding showed that NOTCH-1 exhibited elevated level in EGFR-mutant TKI-resistant NSCLC cells 
A

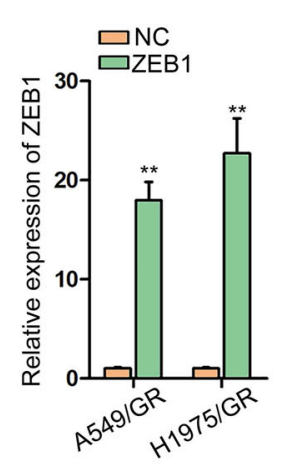

C

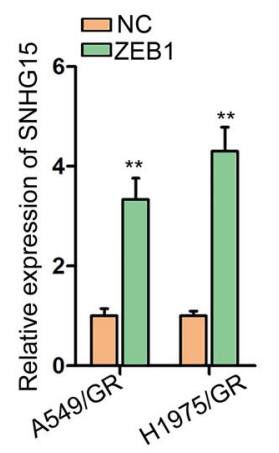

E

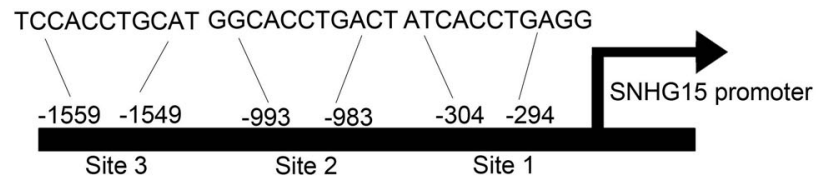

$\mathrm{F}$

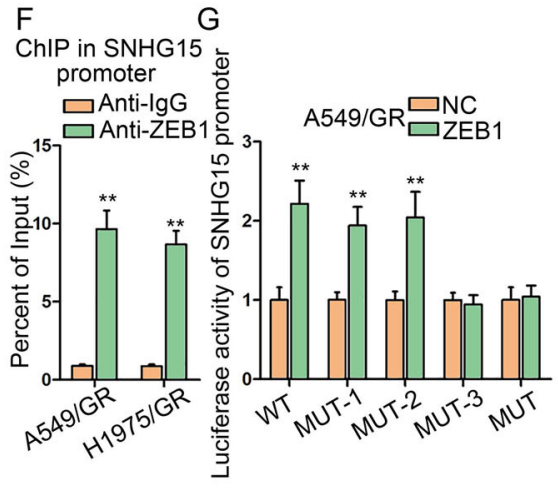

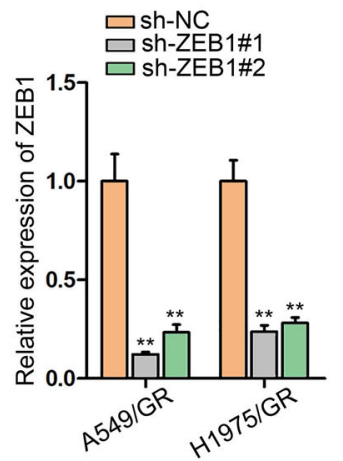

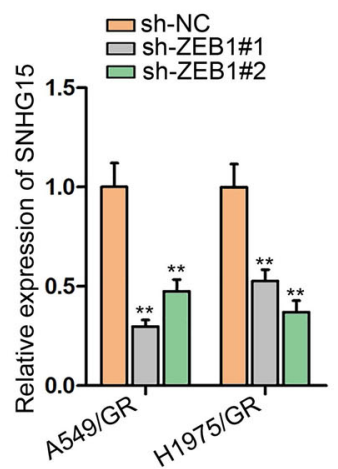

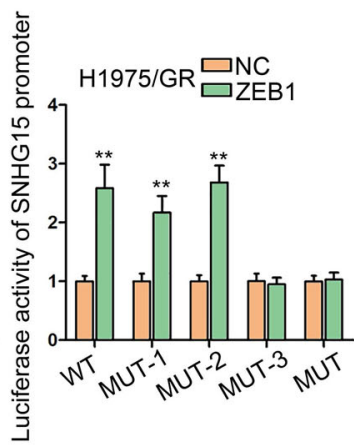

B
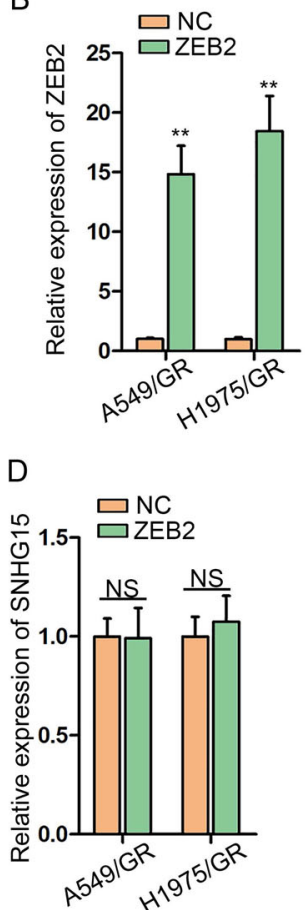
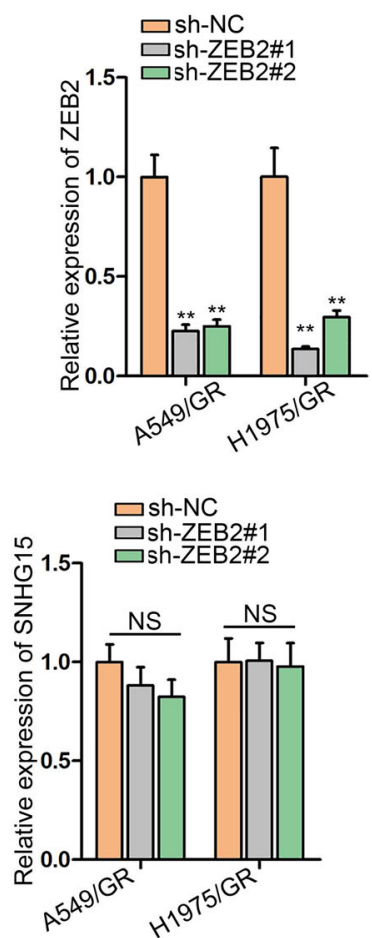

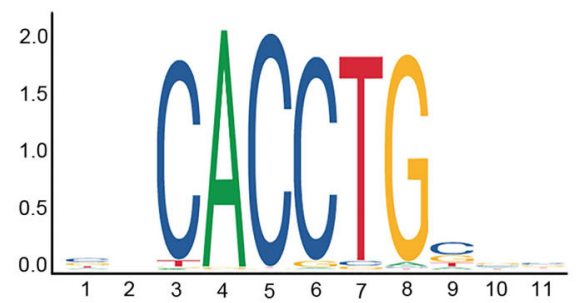

$\mathrm{H}$
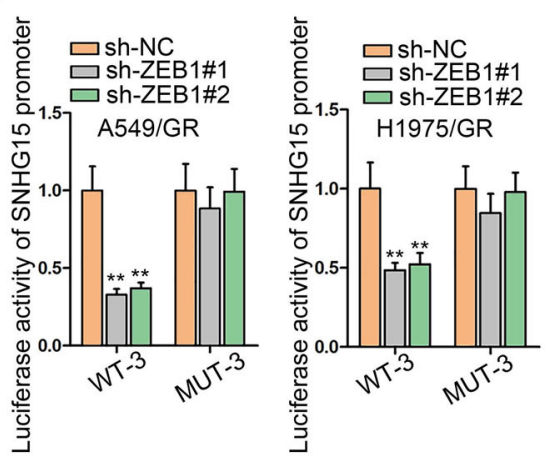

Fig. 6 ZEB1 trans-activates SNHG15 expression in gefitinib-resistant LUAD cells. $\mathbf{a}$, $\mathbf{b}$ qRT-PCR was used to test the expression of ZEB1/2 and inhibition efficiency of ZEB1/2 in A549/GR and H1975/GR. c, d The influence on SNHG15 expression caused by ZEB1/2 knockdown or overexpression was tested by qRT-PCR. e The binding sites between SNHG15 and ZEB1 and sequence logo were presented according to JASPAR (http://jaspar. genereg.net//. $\mathbf{f}$ ChIP assay was conducted to prove that ZEB1 could bind to SNHG15 promoter in A549/GR and H1975/GR. g, h Luciferase reporter assay was conducted to test the binding of ZEB1 at predicted site of SNHG15 promoter in A549/GR and H1975/GR. Data obtained from three replications were shown as mean \pm S.D. ${ }^{* *} P<0.01$ indicated that differences were statistically significant. NS no significance.

compared with the parental sensitive cells ${ }^{39}$, indicating the potential participation of NOTCH-1 in LUAD. Our study applied EGFR wild-type LUAD cells and observed that NOTCH-1 signaling pathway was activated and NOTCH-1 protein level was promoted in A549/GR and H1975/GR cells compared with the parental non-resistant 

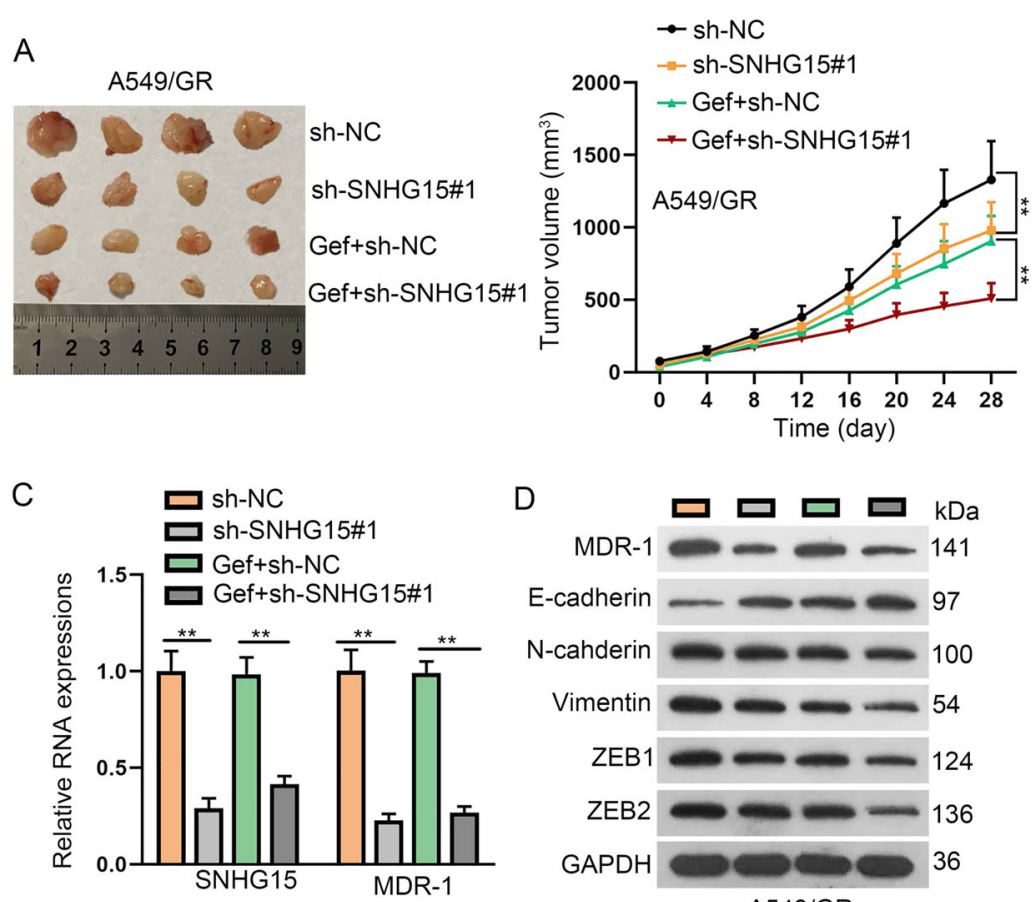

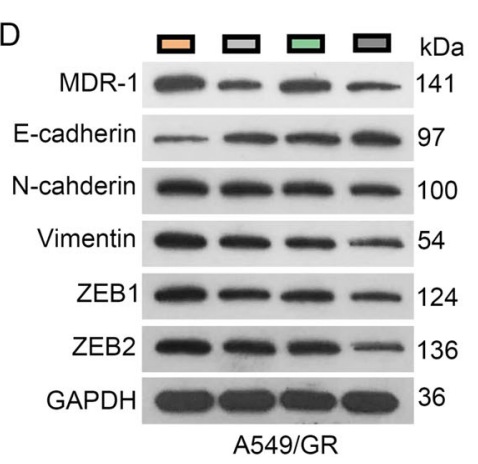

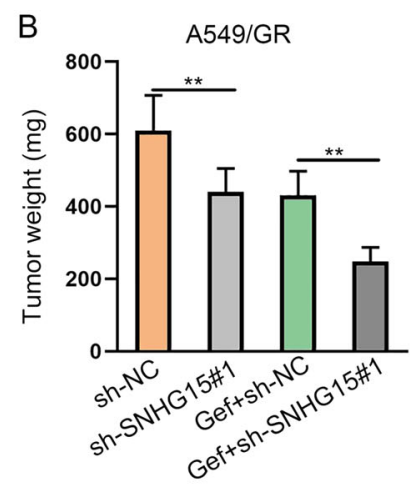

Fig. 7 SNHG15 knockdown reversed gefitinib resistance in LUAD in vivo. a Mice were injected with A549/GR cells transfected with sh-NC or shSNHG15\#1 and then mice were treated with DMSO or gefitinib. Tumor growth was measured every 4 days to draw the growth curve. $\mathbf{b}$ Tumor weight at 28 day was measured in each group. $\mathbf{c}$ qRT-PCR data of SNHG15 and MDR-1 in each group. $\mathbf{d}$ Western blot of MDR-1, E-cadherin, $\mathrm{N}$-cadherin, Vimentin, ZEB1, and ZEB2 in each group. Data obtained from three replications were shown as mean \pm S.D. ${ }^{*} P<0.01$ indicated that differences were statistically significant.

cell lines, confirming the link between NOTCH-1 and TKI resistance in LUAD. In addition, several reports proved that NOTCH-1 positively regulated EGFR in NSCLC cells both in EGFR mutant (PC9 and NCI-H1650 cell lines with EGFR exon 19 deletion (DelE746-A750)) and EGFR WT cell lines (NCI-H520) ${ }^{4,22}$ Herein, our data consistently showed the positive regulation of NOTCH-1 on EGFR expression, indicating that $\mathrm{NOTCH}-1$ might affect resistance to gefitinib in LUAD cells via EGFR signaling.

Long noncoding RNAs (lncRNAs), with a length of over $200 \mathrm{nt}$ and limited protein-coding ability, have been proved to function indispensably in regulating stemness of cancer stem cells and chemoresistance ${ }^{40,41}$. LncRNA CASC9.5 and LINC00312 have been suggested to function as oncogenes in the development of $\operatorname{LUAD}^{42,43}$. Besides, lncRNA GAS5 has been discovered to facilitate gefitinib-triggered cell death in innate EGFR TKI-resistant LUAD cells via reducing IGF-1R level ${ }^{44}$. Existing literature pointed out that SNHG15 could exert oncogenic functions on the progression of colon cancer, NSCLC, colorectal carcinoma, and osteosarcoma via acting as a competing endogenous RNA (ceRNA) or interacting $\mathrm{TFs}^{12,15,45-47}$. What interested us was that SNHG15 has been linked to chemoresistance such as temosolomide resistance in glioma and 5-FU resistance in colorectal cancer $^{14,15}$. However, SNHG15 has never been linked to gefitinib in cancer. Although SNHG15 has been related to lung cancer before ${ }^{48}$, the never has SNHG15 been associated with drug resistance in LUAD. Our data first verified that SNHG15 was downregulated by NOTCH-1 deficiency in gefitinib-resistant LUAD cells. Intriguingly, we found that SNHG15 cannot affect EGFR expression in gefitinib-resistant LUAD cells. This suggested that NOTCH-1 might affect GR via SNHG15 independent from EGFR signaling. Then, it was revealed that absence of SNHG15 induced an inhibition in cell proliferation, cell migration and EMT process as well as a promotion in cell apoptosis in gefitinib-resistant LUAD cells. Collectively, we first manifested that SNHG15 exhibited oncogenic properties in gefitinib-resistant LUAD cells. Also, we confirmed that SNHG15 knockdown overcome GR in vivo.

In recent years, the ceRNA regulatory network constructed by lncRNAs in cancers has drawn the attention of researchers. With the miRNA response elements, lncRNA could compete with mRNA to bind with miRNA, thereby freeing mRNA from the regulation of miRNA ${ }^{49,50}$. This work revealed that SNHG15 was mainly localized in the cytoplasm of A549/GR and H1975/GR cells, implying that 
SNHG15 might function as a ceRNA. Therefore, we searched Starbase and identified that miR-451 was closely related to NOTCH-1 by observing that miR-451 was the most upregulated under NOTCH-1 knockdown among the 13 predicted miRNAs. Our previous paper has elucidated the suppressed level of miR-451 in LUAD cells as well as the functions and molecular mechanism of dysregulated miR-451 on radioresistance of docetaxelresistant LUAD cells ${ }^{19-21}$. In this study, interaction between miR-451 and SNHG15 was first uncovered by our data, and miR-451 was first linked to GR as well. Moreover, miR-451 overexpression was uncovered to restrain cell proliferation, cell migration, and EMT process as well as enhance apoptosis in resistant LUAD cells. We also provided interesting data that miR-451 failed to affect EGFR level in gefitinib-resistant LUAD cells. Former study supported the negative regulation of miR-451 on EGFR level in breast cancer ${ }^{23}$. This inconsistency indicated that the regulation of miR-451 on EGFR might depend on cell type and intracellular context.

Afterwards, MDR-1, which has been found to be regulated by AP-1 to influence chemoresistance of LUAD cells $^{51}$, was unraveled as the target of miR-451. Besides, the affinity between MDR-1 and miR-451 was proved ${ }^{51}$. Moreover, it has been demonstrated that miR-451-MDR-1 was downstream of NOTCH-1 in regulating docetaxelresistant LUAD cells ${ }^{51}$. Our data offered new evidence that NOTCH-1 and miR-451/MDR-1 axis could be linked by SNHG15 in GR. In addition, we showed that MDR-1 cannot affect EGFR expression in gefitinib-resistant LUAD cells. In consistence, an existing study showed that overexpressing MDR1 in breast cancer cells caused no significant change in EGFR level ${ }^{52}$. Functional assays revealed that MDR-1 posed pro-proliferation, pro-migration, proEMT process, and anti-apoptosis effects on gefitinibresistant LUAD cells. In subsequence, rescue assays implied that SNHG regulates the malignancy of gefitinibresistant LUAD cells in a miR-451 and MDR-1 dependent way. It has been well established that TFs could promote or inhibit the expression of genes, including lncRNAs ${ }^{53,54}$. As a key activator of EMT process, ZEB1 has been shown to activate DKK1 transcription and suppress Fbxo32 and Trim63 transcription ${ }^{55,56}$. Herein, we first detected the upstream mechanism of SNHG15 in LUAD. Data from our assays first suggested that SNHG15 could be transcriptionally activated by ZEB1.

This work took the first endeavor to explore the ceRNA role of SNHG15 in gefitinib-resistant LUAD cells. Taken together, NOTCH-1 confers chemoresistance of LUAD cells to gefitinib via SNHG15/miR-451/ZEB1 feedback loop, shedding a new light into the understanding of GR of LUAD cells. The weakness of this study is that the relation of miR451 with MDR-1 has been reported by previous study, and the detailed mechanism whereby SNHG15/miR-451/ZEB1 feedback loop-regulated GR in LUAD cells independent from EGFR will be further explained in the future.

\section{Acknowledgements}

We sincerely appreciate lab members.

Conflict of interest

The authors declare that they have no conflict of interest.

\section{Publisher's note}

Springer Nature remains neutral with regard to jurisdictional claims in published maps and institutional affiliations.

Supplementary Information accompanies this paper at (https://doi.org/ 10.1038/s41419-020-2683-x).

Received: 24 October 2019 Revised: 1 February 2020 Accepted: 3 February 2020

Published online: 13 July 2020

References

1. Jemal, A. et al. Global cancer statistics. CA: A Cancer J. Clinicians 61, 69-90 (2011).

2. Giuliani, J. \& Bonetti, A. Pharmacologic costs of tyrosine kinase inhibitors in first-line therapy for advanced non-small-cell lung cancer with activating epidermal growth factor receptor mutations: a review of pivotal phase III randomized controlled trials. Clin. Lung Cancer 17, 91-94 (2016).

3. Xie, M. et al. Activation of Notch-1 enhances epithelial-mesenchymal transition in gefitinib-acquired resistant lung cancer cells. J. Cell. Biochem. 113, 1501-1513 (2012).

4. Xie, M., He, C. S., Wei, S. H. \& Zhang, L. Notch-1 contributes to epidermal growth factor receptor tyrosine kinase inhibitor acquired resistance in nonsmall cell lung cancer in vitro and in vivo. Eur. J. Cancer 49, 3559-3572 (2013).

5. Huang, J. et al. Expression of Notch-1 and its clinical significance in different histological subtypes of human lung adenocarcinoma. J. Exp. Clin. Cancer Res. 32, 84 (2013).

6. Ibrahim, S. A. et al. Syndecan-1 is a novel molecular marker for triple negative inflammatory breast cancer and modulates the cancer stem cell phenotype via the IL-6/STAT3, Notch and EGFR signaling pathways. Mol. Cancer 16, 57 (2017).

7. Ye, Y. et al. COX-2 regulates Snail expression in gastric cancer via the Notch1 signaling pathway. Int. J. Mol. Med. 40, 512-522 (2017).

8. Zhang, Y. et al. Downregulation of SNHG1 suppresses cell proliferation and invasion by regulating Notch signaling pathway in esophageal squamous cell cancer. Cancer Biomarkers: Sect. A Dis. markers 21, 89-96 (2017).

9. Kornienko, A. E., Guenzl, P. M., Barlow, D. P. \& Pauler, F. M. Gene regulation by the act of long non-coding RNA transcription. BMC Biol. 11, 59 (2013).

10. Chen, D. L. et al. Long noncoding RNA XIST expedites metastasis and modulates epithelial-mesenchymal transition in colorectal cancer. Cell Death Dis. 8, e3011 (2017).

11. Zhang, $M$. et al. Long noncoding RNA CRNDE/PRC2 participated in the radiotherapy resistance of human lung adenocarcinoma through targeting p21 expression. Oncol. Res. 26, 1245-1255 (2018).

12. Jiang, $\mathrm{H}$. et al. Long non-coding RNA SNHG15 interacts with and stabilizes transcription factor Slug and promotes colon cancer progression. Cancer Lett. 425, 78-87 (2018).

13. Du, Y. et al. Knockdown of SNHG15 suppresses renal cell carcinoma proliferation and EMT by regulating the NF-kappaB signaling pathway. Int. J. Oncol. 53, 384-394 (2018).

14. Li, Z. et al. Modulating IncRNA SNHG15/CDK6/miR-627 circuit by palbociclib, overcomes temozolomide resistance and reduces M2-polarization of glioma associated microglia in glioblastoma multiforme. J. Exp. Clin. Cancer Res. 38, 380 (2019).

15. Saeinasab, M. et al. SNHG15 is a bifunctional MYC-regulated noncoding locus encoding a IncRNA that promotes cell proliferation, invasion and drug resistance in colorectal cancer by interacting with AIF. J. Exp. Clin. cancer Res. 38, 172 (2019). 
16. Wang, Y. et al. The long noncoding RNA HULC promotes liver cancer by increasing the expression of the HMGA2 oncogene via sequestration of the microRNA-186. J. Biol. Chem. 292, 15395-15407 (2017).

17. Dong, H. X., Wang, R., Jin, X. Y., Zeng, J. \& Pan, J. LncRNA DGCR5 promotes lung adenocarcinoma (LUAD) progression via inhibiting hsa-mir-22-3p. J. Cell. Physiol. 233, 4126-4136 (2018).

18. Goto, A. et al. The low expression of miR-451 predicts a worse prognosis in non-small cell lung cancer cases. PLoS ONE 12, e0181270 (2017).

19. Wang, R. et al. MicroRNA-451 functions as a tumor suppressor in human nonsmall cell lung cancer by targeting ras-related protein 14 (RAB14). Oncogene 30, 2644-2658 (2011).

20. Chen, D. et al. MicroRNA-451 induces epithelial-mesenchymal transition in docetaxel-resistant lung adenocarcinoma cells by targeting proto-oncogene c-Myc. Eur. J. Cancer 50, 3050-3067 (2014).

21. Wang, R. et al. Acquisition of radioresistance in docetaxel-resistant human lung adenocarcinoma cells is linked with dysregulation of miR-451/c-Myc-survivin/ rad-51 signaling. Oncotarget 5, 6113-6129 (2014).

22. Baumgart, A. et al. ADAM17 regulates epidermal growth factor receptor expression through the activation of Notch1 in non-small cell lung cancer. Cancer Res. 70, 5368-5378 (2010).

23. Bergamaschi, A. \& Katzenellenbogen, B. S. Tamoxifen downregulation of miR451 increases $14-3-3 \zeta$ and promotes breast cancer cell survival and endocrine resistance. Oncogene 31, 39-47 (2012).

24. Goossens, S. et al. ZEB2 and LMO2 drive immature T-cell lymphoblastic leukemia via distinct oncogenic mechanisms. Haematologica https:/doi.org/ 10.3324/haematol.2018.207837 (2019).

25. Du, X. et al. LINC00511 contributes to glioblastoma tumorigenesis and epithelial-mesenchymal transition via LINC00511/miR-524-5p/YB1/ZEB1 positive feedback loop. J. Cell. Mol. Med. https://doi.org/10.1111/jcmm.14829 (2019).

26. Zhao, L., Liu, Y., Zhang, J., Liu, Y. \& Qi, Q. LnCRNA SNHG14/miR-5590-3p/ZEB1 positive feedback loop promoted diffuse large B cell lymphoma progression and immune evasion through regulating PD-1/PD-L1 checkpoint. Cell Death Dis. 10, 731 (2019).

27. Ohashi, K., Maruvka, Y. E., Michor, F. \& Pao, W. Epidermal growth factor receptor tyrosine kinase inhibitor-resistant disease. J. Clin. Oncol. 31, 1070-1080 (2013).

28. Ninomiya, $\mathrm{K}$. et al. MET or NRAS amplification is an acquired resistance mechanism to the third-generation EGFR inhibitor naquotinib. Sci. Rep. 8 1955 (2018).

29. Sequist, L. V. et al. Phase III study of afatinib or cisplatin plus pemetrexed in patients with metastatic lung adenocarcinoma with EGFR mutations. J. Clin Oncol. 31, 3327-3334 (2013)

30. Soria, J. C. et al. Osimertinib in untreated EGFR-mutated advanced non-smallcell lung cancer. N. Engl. J. Med. 378, 113-125 (2018).

31. Soon, Y. Y., Leong, C. N., Koh, W. Y. \& Tham, I. W. EGFR tyrosine kinase inhibitors versus cranial radiation therapy for EGFR mutant non-small cell lung cancer with brain metastases: a systematic review and meta-analysis. Radiother. Oncol. 114, 167-172 (2015).

32. Mansour, M. R. et al. Notch-1 mutations are secondary events in some patients with T-cell acute lymphoblastic leukemia. Clin. Cancer Res. 13, 6964-6969 (2007).

33. Jundt, F. et al. Aberrant expression of Notch1 interferes with the B-lymphoid phenotype of neoplastic B cells in classical Hodgkin lymphoma. Leukemia 22, 1587-1594 (2008)

34. Purow, B. W. et al. Expression of Notch-1 and its ligands, Delta-like-1 and Jagged-1, is critical for glioma cell survival and proliferation. Cancer Res. $\mathbf{6 5}$ 2353-2363 (2005)

35. Dalrymple, S. et al. Role of notch-1 and E-cadherin in the differential response to calcium in culturing normal versus malignant prostate cells. Cancer Res. $\mathbf{6 5}$, 9269-9279 (2005)

36. Donnem, T. et al. Prognostic impact of Notch ligands and receptors in nonsmall cell lung cancer: coexpression of Notch-1 and vascular endothelial growth factor-A predicts poor survival. Cancer $\mathbf{1 1 6}$ 5676-5685 (2010).

37. Kang, J. et al. Rhamnetin and cirsiliol induce radiosensitization and inhibition of epithelial-mesenchymal transition (EMT) by miR-34a-mediated suppression of Notch-1 expression in non-small cell lung cancer cell lines. J. Biol. Chem. 288, 27343-27357 (2013)

38. Eliasz, S. et al. Notch-1 stimulates survival of lung adenocarcinoma cells during hypoxia by activating the IGF-1R pathway. Oncogene 29, 2488-2498 (2010)

39. Xie, M., He, C.-S., Wei, S.-H. \& Zhang, L. Notch-1 contributes to epidermal growth factor receptor tyrosine kinase inhibitor acquired resistance in nonsmall cell lung cancer $<$ em $>$ in vitro $</$ em $>$ and $<$ em $>$ in vivo $</$ em $>$. Eur. J. Cancer 49, 3559-3572 (2013).

40. Yan, H. \& Bu, P. Non-coding RNAs in cancer stem cells. Cancer Lett. 421 $121-126$ (2018)

41. Zheng, Y., Liu, L. \& Shukla, G. C. A comprehensive review of web-based non-coding RNA resources for cancer research. Cancer Lett. 407, 1-8 (2017).

42. Zhou, J. et al. Long noncoding RNA CASC9.5 promotes the proliferation and metastasis of lung adenocarcinoma. Sci. Rep. 8, 37 (2018).

43. Peng, Z. et al. The long noncoding RNA LINC00312 induces lung adenocarcinoma migration and vasculogenic mimicry through directly binding YBX1. Mol. Cancer 17, 167 (2018).

44. Dong, S. et al. The long non-coding RNA, GAS5, enhances gefitinib-induced cell death in innate EGFR tyrosine kinase inhibitor-resistant lung adenocarcinoma cells with wide-type EGFR via downregulation of the IGF-1R expression. J. Hematol. Oncol. 8, 43 (2015).

45. Sun, X. et al. Long noncoding RNA SNHG15 enhances the development of colorectal carcinoma via functioning as a ceRNA through miR-141/ SIRT1/Wnt/beta-catenin axis. Artif. Cells, Nanomed., Biotechnol. 47 2536-2544 (2019).

46. Liu, K., Hou, Y., Liu, Y. \& Zheng, J. LncRNA SNHG15 contributes to proliferation, invasion and autophagy in osteosarcoma cells by sponging miR-141. J. Biomed. Sci. 24, 46 (2017).

47. Xue, M. et al. HOTAIR induces the ubiquitination of Runx3 by interacting with Mex3b and enhances the invasion of gastric cancer cells. Gastric Cancer 21, 756-764 (2018).

48. Jin, B., Jin, H., Wu, H. B., Xu, J. J. \& Li, B. Long non-coding RNA SNHG15 promotes CDK14 expression via miR-486 to accelerate non-small cell lung cancer cells progression and metastasis. J. Cell. Physiol. https://doi.org/10.1002/ jcp.26543 (2018).

49. Dykes, I. M. \& Emanueli, C. Transcriptional and post-transcriptional gene regulation by long non-coding RNA. Genomics, Proteom. Bioinforma. 15, 177-186 (2017).

50. Qi, X. et al. ceRNA in cancer: possible functions and clinical implications. J. Med Genet. 52, 710-718 (2015)

51. Huang, J. et al. Notch-1 confers chemoresistance in lung adenocarcinoma to taxanes through AP-1/microRNA-451 mediated regulation of MDR-1. Mol. Ther. Nucleic Acids 5, e375 (2016).

52. Clarke, R. et al. Effect of P-glycoprotein expression on sensitivity to hormones in MCF-7 human breast cancer cells. J. Natl Cancer Inst. 84 1506-1512 (1992)

53. Gordon, S., Akopyan, G., Garban, H. \& Bonavida, B. Transcription factor YY1: structure, function, and therapeutic implications in cancer biology. Oncogene 25, 1125-1142 (2006)

54. Xu, P., Lin, W., Liu, F., Tartakoff, A. \& Tao, T. Competitive regulation of IPO4 transcription by ELK1 and GABP. Gene 613, 30-38 (2017).

55. Ninfali, C., Siles, L., Darling, D. S. \& Postigo, A. Regulation of muscle atrophyrelated genes by the opposing transcriptional activities of ZEB1/CtBP and FOXO3. Nucleic acids Res. 46, 10697-10708 (2018)

56. de Barrios, O. et al. ZEB1-induced tumourigenesis requires senescence inhibition via activation of DKK1/mutant p53/Mdm2/CtBP and repression of macroH2A1. Gut 66, 666-682 (2017). 